\title{
Quality Evaluation and Pattern Recognition Analyses of Bioactive Marker Compounds from Farfarae Flos Using HPLC/PDA
}

\author{
U Min Seo, ${ }^{a}$ Bing Tian Zhao, ${ }^{a}$ Won Il Kim, ${ }^{a}$ Eun Kyoung Seo, ${ }^{b}$ Jae Hyun Lee, ${ }^{c}$ Byung Sun Min, ${ }^{a}$ \\ Beom Soo Shin, ${ }^{a}$ Jong Keun Son, ${ }^{d}$ and Mi Hee Woo*,a \\ ${ }^{a}$ College of Pharmacy, Catholic University of Daegu; Gyeongsan 712-702, Republic of Korea: ${ }^{b}$ College of \\ Pharmacy and Division of Life and Pharmaceutical Sciences, Ewha Womans University; Seoul 120-750, Republic of \\ Korea: ${ }^{c}$ College of Oriental Medicine, Dongguk University; Gyeongju 780-714, Korea: and ${ }^{d}$ College of Pharmacy, \\ Yeungnam University; Gyeongsan 712-749, Republic of Korea. \\ Received March 5, 2015; accepted April 22, 2015; advance publication released online May 14, 2015
}

The flower bud of Tussilago farfara L., called Farfarae Flos, has traditionally been used in Oriental medicine for the treatment of bronchitis and asthma. To establish a standard for quality control as well as the reliable identification of Farfarae Flos, the contents of five standards, rutin (1), isoquercetin (2), 3,5-dicaffeoylquinic acid (3), tussilagone (4), and tussilagonone (5), were determined by quantitative high-performance liquid chromatography (HPLC)/photodiode array (PDA) analysis. The five standards were separated on a YoungJinBioChrom Aegispak C18-L $(250-\mathrm{mm} \times 4.6-\mathrm{mm}, 5-\mu \mathrm{m})$ column by gradient elution using $0.03 \%$ trifluoroacetic acid in water (A), with acetonitrile (B) as the mobile phase. The flow rate was $1.0 \mathrm{~mL} / \mathrm{min}$, and the UV detector wavelength was set at $220 \mathrm{~nm}$. The method was successfully used in the analysis of Farfarae Flos from different geographic origins with relatively simple conditions and procedures, and the results demonstrated satisfactory linearity, recovery, precision, accuracy, stability, and robustness. The HPLC analytical method for pattern recognition analysis was validated by repeated analysis of 62 Farfarae Flos samples. This result indicated that the established HPLC/PDA method is suitable for quantitation and pattern recognition analyses for the quality evaluation of Farfarae Flos.

Key words Farfarae Flos; HPLC; pattern recognition; quality control

Herbal medicines have a long history of use in therapeutic fields, and are attracting considerable attention because of their low toxicity and excellent therapeutic benefits. Quality control of herbal medicines is more difficult than synthetic drugs due to the chemical complexity of the material, wherein a single active substance is not responsible for the overall pharmacological potency. Pattern recognition analysis of quality control for traditionally used herbs can provide information on the overall chemical composition.) Tussilago farfara L., one of the Chinese traditional medicines, is widely spread in China, North Africa, Siberia, and Europe. The flower buds of T. farfara L. (Compositae) are called Farfarae Flos, and have been used for the treatment of cough, bronchitis, and asthmatic disorders in Asia. ${ }^{2,3)}$ This bud, also known as Kuan-Dong-Hua in China, is a popular folk medicine, and has been studied for its pharmacological activities, including anti-oxidative, anti-microbial, anti-inflammatory, anti-diabetic, anti-hypertension, platelet anti-aggregation, and anti-cancer functions. ${ }^{4-8)}$

Farfarae Flos is the flower bud of T. farfara L. in the Chinese Pharmacopoeia (C.P. 2010) and the Korean Pharmacopoeia (K.P. 2014). ${ }^{9,10)}$ The regulation of Farfarae Flos content in the C.P. was already stipulated in 2010, which prescribed that more than $0.07 \%$ tussilagone from Farfarae Flos must be included. However, the K.P. has no stipulations on the main compounds contained in Farfarae Flos. Therefore, the purpose of this study was to establish a reliable HPLC method for the quantitative analysis of the major compounds in Farfarae Flos, and to provide analytical methods which can be used as the official analytical method of the K.P.

Previously, a few researchers quantified only the amount of rutin, one of the bioactive components in Farfarae Flos. ${ }^{11-13)}$ However, they ignored other reasonable component, such as tussilagone. $\mathrm{Wu}$ et al. established a method for the quantitative analysis of rutin, isoquercitrin, and chlorogenic acid in $50 \mathrm{~min}$ at $255 \mathrm{~nm}$ using three solvent system such as methanol, acetonitrile, and $0.2 \%$ formic acid in water. ${ }^{14)}$ Zhang et al. developed a reverse phase (RP)-HPLC method for the simultaneous determination of chlorogenic acid and rutin in Farfarae Flos by HPLC with an RP-C18 column and a UV detector at $254 \mathrm{~nm}$, but this method was unreliable, because the first compound (chlorogenic acid) came out too early, near the solvent peak at about 5 min. ${ }^{15)}$ Ma et al. later established a method for the quantitative analysis of the quercetin and kaempferide in Farfarae Flos, in which two marker compounds were not considered as characteristic components. ${ }^{16)}$ As was stated above, their studies were focused on the quantification of only flavonoid and phenolic compounds without unique alkaloid components such as tussilagone and tussilagonone in Farfarae Flos.

In this study, the developed method not only had short analytical time, but also showed good resolution. An HPLC/photodiode array (PDA) method was established for analysis of rutin, isoquercetin, 3,5-dicaffeoylquinic acid, tussilagone, and tussilagonone as marker compounds. The methods for plant extraction and HPLC analysis were optimized and validated. A calibration curve of the marker compounds was also made, and variations of the retention times according to the $\mathrm{pH}$ of the mobile phase as well as the stability of marker compounds in solution were investigated. We therefore suggest a suitable analytical method for quantitative and pattern recognition analyses of Farfarae Flos, together with the establishment of adequate marker compounds [rutin (1), isoquercetin (2), 
3,5-dicaffeoylquinic acid (3), tussilagone (4), and tussilagonone (5)] to distinguish between Chinese and Korean Farfarae Flos.

\section{Experimental}

Materials All of the standard compounds were provided by Prof. Eun Kyoung Seo, Ewha Womans University, Seoul, Korea. Their structures were unambiguously identified as rutin (1), isoquercetin (2), 3,5-dicaffeoylquinic acid (3), tussilagone (4), and tussilagonone (5). The standard compound structures are shown in Fig. 1. Purity of standard compounds was estimated to be higher than $95 \%$ based on HPLC analysis. Internal standard, amiodarone hydrochloride, was purchased from Sigma Chemicals (St. Louis, MO, U.S.A.). Acetonitrile was purchased from Merck K GaA (Darmstadt, Germany). All other chemicals used were of analytical grade unless otherwise noted. Distilled water was prepared using the Milli-Q purification system (Millipore, Bedford, MA, U.S.A.). Sixtytwo samples cultured in different regions were provided from Prof. Jae-Hyun Lee, College of Oriental Medicine, Dongguk University at Gyeongju, Korea. This study adopted the following sixty-two samples corresponding to Farfarae Flos [six ones (K01-K06) from Korea and fifty-six ones (C07-C62) from China].

Sample Preparation The dried Farfarae Flos was pulverized and passed through a 50 mesh sieve. About $2.0 \mathrm{~g}$ of the powder, accurately weighed, were added to $40 \mathrm{~mL}$ of $95 \%$ methanol containing an internal standard (I.S.: $50 \mathrm{ppm}$ amio- darone hydrochloride) by sonication for $60 \mathrm{~min}$ at room temperature. After extraction, each sample solution was adjusted to the original volume and filtered through $0.45 \mu \mathrm{m}$ membrane, and an aliquot $(10 \mu \mathrm{L})$ of the filtrate was injected into HPLC.

HPLC/PDA Conditions The HPLC equipment was a Waters 2695 Separations Module (Waters, Milford, MA, U.S.A.) with Waters 996 Photodiode Array Detector. YoungJinBioChrom Aegispak C18-L $(250 \mathrm{~mm} \times 4.6 \mathrm{~mm}$, $5 \mu \mathrm{m})$, Phenomenex Gemini C18 110A $(250 \mathrm{~mm} \times 4.6 \mathrm{~mm}$, $5 \mu \mathrm{m})$ and Shiseido $\mathrm{C} 18(250 \mathrm{~mm} \times 4.6 \mathrm{~mm}, 5 \mu \mathrm{m})$ columns were tested with the guard column $(3.9 \mathrm{~mm} \times 20 \mathrm{~mm}$ XTerra $\mathrm{RP} 18,5 \mu \mathrm{m})$ filled with the same stationary phase. The mobile phase consisted of water containing $0.03 \%$ trifluoroacetic acid (A) and acetonitrile (B). Elution was performed at a flow rate of $1.0 \mathrm{~mL} / \mathrm{min}$ in gradient and isocratic modes. The solvent gradient was changed according to the following program: [0-15 min, $15-30 \%$ (B); $15-20 \mathrm{~min}, 30-73 \%$ (B) and $20-55 \mathrm{~min}, 73 \%$ (B)]. The column was washed by $100 \% \mathrm{~B}$ for $20 \mathrm{~min}$. And each run was followed by a $20 \mathrm{~min}$ re-equilibration. Equilibration time for the next run is $20 \mathrm{~min}$. The mobile phase was filtered under vacuum through a $0.21-\mu \mathrm{m}$ membrane filter and degassed prior to use. The analysis was carried out at a flow rate of $1.0 \mathrm{~mL} / \mathrm{min}$ with PDA detection absorbance at $220 \mathrm{~nm}$. The chromatograms were processed using Empower pro software, Build 1154 (Waters).

Analytical Method Validation The standards of rutin (1), isoquercetin (2), 3,5-dicaffeoylquinic acid (3), tussilagone (4), and tussilagonone (5) were each accurately weighed and then<smiles>O=c1c(OC2OC3OC4OC(C(O)C4O)C(O)C3OC2O)c(-c2ccc(O)c(O)c2)oc2cc(O)cc(O)c12</smiles>

Rutin (1)<smiles>Cc1ccc(/C=C/C(=O)O[C@@H]2C[C@](O)(C(=O)O)C[C@H](OC(=O)/C=C/c3ccc(O)c(O)c3)[C@H]2O)cc1O</smiles>

3,5-Dicaffeoylquinic acid (3)

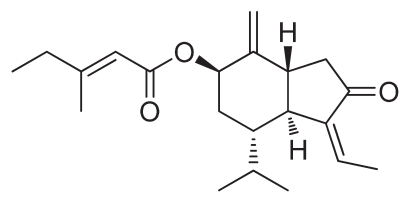

Tussilagonone (5)<smiles>O=c1c(OC2OC(CO)C(O)C(O)C2O)c(-c2ccc(O)c(O)c2)oc2cc(O)cc(O)c12</smiles>

Isoquercetin (2)<smiles>C=C1[C@H]2CC(=O)[C@H](C(C)O[C@H](C)O)[C@@H]2[C@@H](C(C)C)C[C@H]1OC(=O)/C=C(\C)CC</smiles>

Tussilagone (4)<smiles>CCCCc1oc2ccccc2c1C(=O)c1cc(I)c(OCCN(CCC)CCC)c(I)c1</smiles>

Amiodarone hydrochloride (I.S.)

Fig. 1. Structures of Standards and an Internal Standard 
dissolved with 95\% methanol containing I.S. (50 ppm amiodarone hydrochloride) to produce stock standard solutions of $500 \mathrm{ppm}$. The calibration curves were generated after diluting the stock solution with $95 \%$ methanol containing I.S. (50 ppm amiodarone hydrochloride). A reference solution at concentrations of $0.625-500.0 \mu \mathrm{g} / \mathrm{mL}$ was analyzed by HPLC/PDA. The regression equations were calculated in the form of $y=\mathrm{a} x+\mathrm{b}$, where $y$ and $x$ correspond to peak ratio (compound area/I.S. area) and compound concentration, respectively. Limits of detection (LOD) and limits of quantification (LOQ) were obtained based on the concentrations at three times signal-tonoise value $(3=S / N)$ and ten times $(10=S / N)$, respectively.

Recovery tests were executed by mixing a powdered sample $(2.0 \mathrm{~g})$ of the reference compounds at three control levels (near the LOQ, medium and higher concentrations from the calibration curves). The mixture was then extracted by sonication in $40 \mathrm{~mL}$ of $95 \%$ methanol containing I.S. (50 ppm amiodarone hydrochloride) at room temperature $\left(25^{\circ} \mathrm{C}\right)$ for $60 \mathrm{~min}$. The extract solution was filtered through a $0.45-\mu \mathrm{m}$ membrane. The HPLC/PDA analysis experiments were performed in triplicate for each control level. The data from the standard solution and the extracted sample were compared.

Precision and accuracy were determined by multiple analyses $(n=5)$ of quality control samples prepared at low, medium and high concentrations spanning the calibration range.

The robustness of the method was studied by introducing changes in columns (i.e., Aegispak, Gemini and Shiseido), separation temperatures (i.e., $25,30,35^{\circ} \mathrm{C}$ ) and three different flow rates (i.e., $0.8,1.0,1.2 \mathrm{~mL} / \mathrm{min}$ ).

Stability was tested with mixed standard solution stored in the dark at $4^{\circ} \mathrm{C}$ and room temperature $\left(25^{\circ} \mathrm{C}\right)$. The two samples were analyzed in triplicate at $0,1,3,7,15$, and $30 \mathrm{~d}$.
Pattern Recognition Analysis A pattern recognition analysis was conducted to evaluate the phytochemical equivalency among the 62 Farfarae Flos samples. We used five major marker compounds [rutin (1), isoquercetin (2), 3,5-dicaffeoylquinic acid (3), tussilagone (4), and tussilagonone (5)] for the pattern recognition analysis using IBM SPSS Statistics Version 19 software (SPSS, Inc., Chicago, IL, U.S.A.).

\section{Results and Discussion}

Optimization of Chromatographic Conditions HPLC conditions were selected to obtain good resolution on the chromatograms within a short retention time. For optimization of the chromatographic conditions, the effects of the composition of the mobile phase on separation were examined. A mobile phase of water-methanol proved unsatisfactory for separating the structurally similar compounds. Acetonitrile, as an organic modifier, demonstrated significantly improved separation. We also tested the addition of $0.03,0.1$, and $1 \%$ acid (acetic acid, formic acid, and trifluoroacetic acid) to the mobile phase. The addition of $0.03 \%$ trifluoroacetic acid resulted in good resolution of all compounds, as well as satisfactory peak symmetry and shape. The extracted sample was scanned between 200-400 nm using a PDA detector to determine the appropriate detection wavelength. The reasonable components, such as tussilagone and tussilagonone, showed maximum absorption at $220 \mathrm{~nm}$. Besides these, rutin, isoquercetin, and 3,5-dicaffeoylquinic acid could also be detected well at about $220 \mathrm{~nm}$. $\mathrm{Wu}$ et al. had reported LOD value of rutin as $200 \mathrm{ng} / \mathrm{mL}$ at $255 \mathrm{~nm}$, exhibiting about 20 times higher than our LOD value $(8.58 \mathrm{ng} / \mathrm{mL})$ of rutin at $\left.220 \mathrm{~nm}^{14}\right)$ Therefore, our method using the detection wavelength at $220 \mathrm{~nm}$ is more sensitive than the analytical method at $255 \mathrm{~nm}$. Therefore, all components could
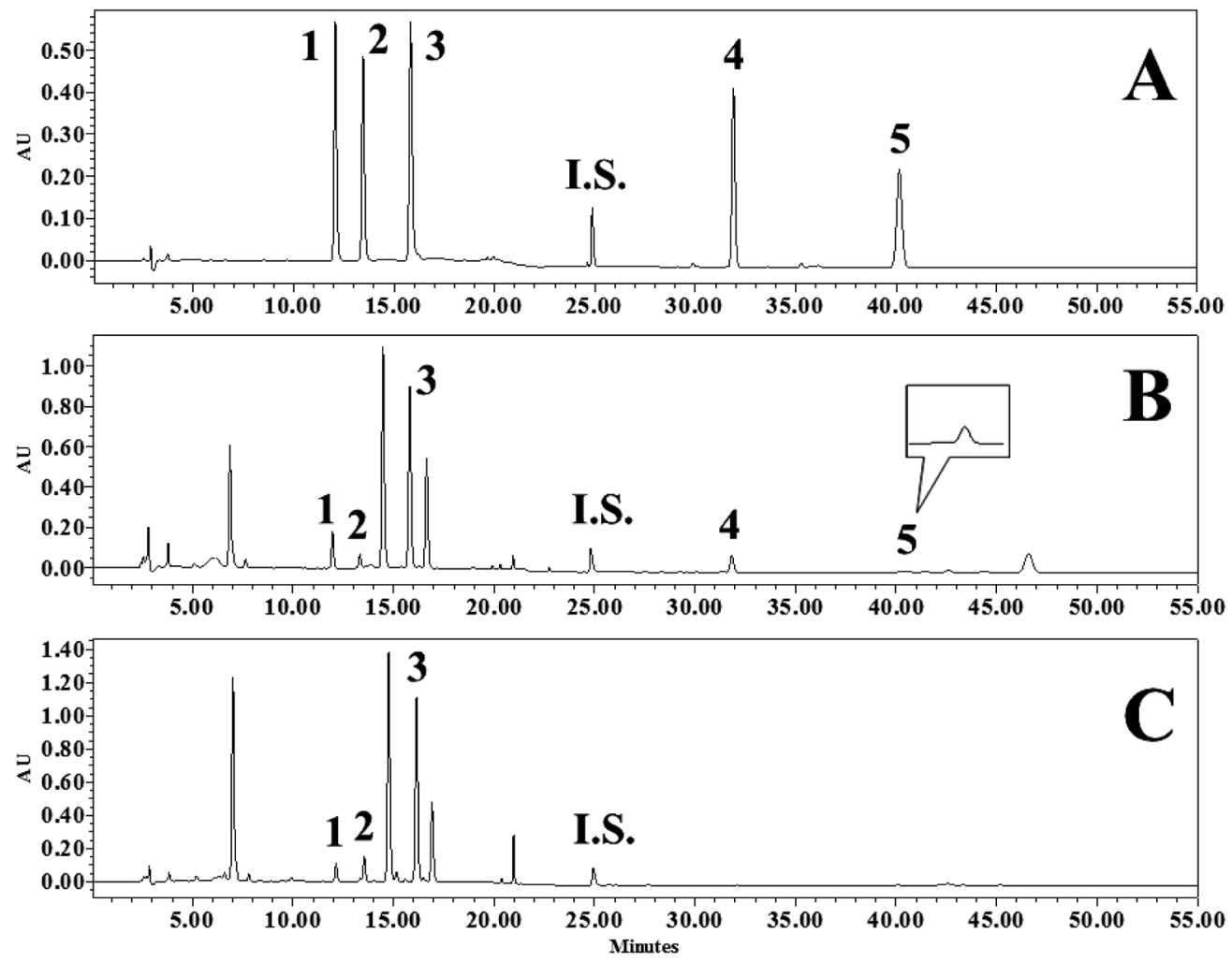

Fig. 2. The HPLC Chromatograms of Standards Mixture (A) and Farfarae Flos Samples [Chinese (B), and Korean (C)]

Rutin (1), isoquercetin (2), 3,5-dicaffeoylquinic acid (3), tussilagone (4), and tussilagonone (5). 
be shown with baseline separation at $220 \mathrm{~nm}$ (data not shown). The typical chromatograms of the sample and standard mixtures are shown in Fig. 2, from which it can be seen that all target compound peaks and internal standard peak were clearly separated within $45 \mathrm{~min}$. Amiodarone hydrochloride was selected as an internal standard. The chromatographic peaks of the analytes in the sample solution were identified by comparing their retention times with those of the reference standards, and were further confirmed by spiking the samples with the reference compounds.

Optimization of Sample Preparation Conditions Six solvents for extraction, including 95\% ethanol, $75 \%$ ethanol, 50\% ethanol, 95\% methanol, 75\% methanol, and 50\% methanol, were compared in sample assays after extraction by sonication for $1 \mathrm{~h}$ at room temperature. When the extraction was carried out with 95\% methanol, the sample assay (compound area/I.S. area) showed higher results than obtained by the other solvent samples. Thus, 95\% methanol was used as the extraction solvent throughout this study. Two extraction methods, ultra-sonication and reflux using 95\% methanol extraction solvent, were also compared. The sample assay results were superior after extraction with sonication than with reflux. To determine the time needed for complete extraction, extraction was carried out for five different lengths of time (30, 45, $60,75,90 \mathrm{~min})$ in $95 \%$ methanol via sonication at room temperature. The extraction time of $60 \mathrm{~min}$ yielded similar sample assay results as $75 \mathrm{~min}$. Therefore, the compounds were thoroughly extracted when the extraction time was $60 \mathrm{~min}$ (data not shown).

Validation of the Method Each coefficient of correlation $\left(r^{2}\right)$ was $>0.999$, as determined by least square analysis, suggesting good linearity between the peak area ratio and the compound concentrations. The LOD and LOQ were evaluated at signal-to-noise $(S / N)$ ratios of 3 and 10, respectively. The LOD and LOQ obtained under our experimental conditions are listed in Table 1. All LOD and LOQ values obtained for these five standards were low enough to allow detection of traces of these compounds in either a crude extract or its preparation.

Precision and accuracy were determined by multiple analysis $(n=5)$ of quality control samples prepared at lower, medium and higher concentrations $(1,50,100 \mu \mathrm{g} / \mathrm{mL})$ spanning the calibration range. Intra-assay precision and accuracy were determined from multiple analyses $(n=5)$ of the quality control samples, carried out within the same analytical run. The quality control samples had intra-assay precision of $<4.18 \%$ and accuracy of 90.9-106.7\%. Inter-assay precision and accuracy were evaluated from multiple analyses $(n=5)$ of the quality control samples, analyzed in a single analytical run for five consecutive days. The quality control samples had an inter-assay precision of $<4.42 \%$ and accuracy of $94.9-109.7 \%$. Thus, the method developed was highly reproducible. The pre-

Table 1. Linearity, Linear Ranges, LOD, and LOQ

\begin{tabular}{|c|c|c|c|c|c|c|}
\hline Analytes & $\begin{array}{l}\text { Linear range } \\
(\mu \mathrm{g} / \mathrm{mL})\end{array}$ & Slope & Intercept & $\begin{array}{c}\text { Correlation } \\
\text { coefficient }\left(r^{2}\right)\end{array}$ & $\begin{array}{c}\mathrm{LOD} \\
(\mathrm{ng} / \mathrm{mL})\end{array}$ & $\begin{array}{c}\text { LOQ } \\
(\mathrm{ng} / \mathrm{mL})\end{array}$ \\
\hline 1 & $0.625-500$ & 0.0220 & 0.0157 & 0.9998 & 8.58 & 28.61 \\
\hline 2 & $0.625-500$ & 0.0197 & 0.0111 & 0.9998 & 10.22 & 34.08 \\
\hline 3 & $0.625-500$ & 0.0249 & -0.0182 & 0.9996 & 11.04 & 36.79 \\
\hline 4 & $0.625-500$ & 0.0237 & 0.0191 & 0.9998 & 7.04 & 23.47 \\
\hline 5 & $0.625-500$ & 0.0223 & 0.0002 & 0.9997 & 12.65 & 42.17 \\
\hline
\end{tabular}

Rutin (1), isoquercetin (2), 3,5-dicaffeoylquinic acid (3), tussilagone (4), and tussilagonone (5).

Table 2. The Results of Precision and Accuracy

\begin{tabular}{|c|c|c|c|c|c|c|c|c|c|c|c|}
\hline \multirow[b]{2}{*}{ Analyte } & \multirow{2}{*}{$\begin{array}{c}\text { Fortified } \\
\text { conc. } \\
(\mu \mathrm{g} / \mathrm{mL})\end{array}$} & \multirow{2}{*}{$\begin{array}{c}\text { Sample } \\
\text { conc. } \\
(\mu \mathrm{g} / \mathrm{mL})\end{array}$} & \multicolumn{4}{|c|}{ Intra-day $(n=5)$} & \multirow{2}{*}{$\begin{array}{c}\text { Sample } \\
\text { conc. } \\
(\mu \mathrm{g} / \mathrm{mL})\end{array}$} & \multicolumn{4}{|c|}{ Inter-day $(n=5)$} \\
\hline & & & $\begin{array}{c}\text { Observed } \\
(\mu \mathrm{g} / \mathrm{mL})\end{array}$ & S.D. & $\begin{array}{c}\text { Accuracy } \\
(\%)\end{array}$ & $\begin{array}{l}\text { Precision } \\
\quad(\%)\end{array}$ & & $\begin{array}{c}\text { Observed } \\
(\mu \mathrm{g} / \mathrm{mL})\end{array}$ & S.D. & $\begin{array}{c}\text { Accuracy } \\
(\%)\end{array}$ & $\begin{array}{c}\text { Precision } \\
\quad(\%)\end{array}$ \\
\hline \multirow{3}{*}{1} & 1 & 36.48 & 37.39 & 1.65 & 91.30 & 1.81 & 36.72 & 37.72 & 1.65 & 99.72 & 1.66 \\
\hline & 50 & 36.48 & 87.28 & 1.73 & 101.61 & 1.70 & 36.72 & 88.11 & 0.89 & 102.79 & 0.86 \\
\hline & 100 & 36.48 & 130.81 & 1.25 & 94.34 & 1.32 & 36.72 & 130.85 & 1.27 & 94.13 & 1.35 \\
\hline \multirow{3}{*}{2} & 1 & 33.07 & 34.05 & 1.56 & 98.12 & 1.59 & 32.91 & 34.01 & 1.95 & 109.70 & 1.78 \\
\hline & 50 & 33.07 & 84.04 & 1.17 & 101.95 & 1.15 & 32.91 & 84.39 & 0.81 & 102.97 & 0.79 \\
\hline & 100 & 33.07 & 126.77 & 3.11 & 93.70 & 3.32 & 32.91 & 127.18 & 3.46 & 94.27 & 3.67 \\
\hline \multirow{3}{*}{3} & 1 & 249.42 & 250.45 & 1.70 & 102.77 & 1.65 & 249.87 & 250.82 & 4.22 & 95.35 & 4.42 \\
\hline & 50 & 249.42 & 299.37 & 2.52 & 99.90 & 2.52 & 249.87 & 299.53 & 4.22 & 99.32 & 4.24 \\
\hline & 100 & 249.42 & 356.13 & 4.46 & 106.71 & 4.18 & 249.87 & 356.87 & 4.65 & 107.00 & 4.35 \\
\hline \multirow{3}{*}{4} & 1 & 22.85 & 23.81 & 1.65 & 96.39 & 1.72 & 22.95 & 23.93 & 2.02 & 98.51 & 2.05 \\
\hline & 50 & 22.85 & 69.51 & 1.24 & 93.33 & 1.33 & 22.95 & 70.18 & 1.17 & 94.46 & 1.24 \\
\hline & 100 & 22.85 & 122.65 & 3.39 & 99.80 & 3.40 & 22.95 & 122.65 & 3.52 & 99.71 & 3.53 \\
\hline \multirow{3}{*}{5} & 1 & 8.21 & 9.12 & 1.57 & 90.87 & 1.73 & 8.00 & 8.95 & 1.66 & 94.91 & 1.74 \\
\hline & 50 & 8.21 & 59.32 & 1.97 & 102.23 & 1.92 & 8.00 & 58.65 & 1.73 & 101.31 & 1.71 \\
\hline & 100 & 8.21 & 110.65 & 2.37 & 102.45 & 2.32 & 8.00 & 109.65 & 2.38 & 101.65 & 2.34 \\
\hline
\end{tabular}

Rutin (1), isoquercetin (2), 3,5-dicaffeoylquinic acid (3), tussilagone (4), and tussilagonone (5). 
cision and accuracy data are presented in Table 2 .

The extraction recovery test was performed by extracting a known amount of the five marker compounds from sample C01. Known amounts of each standard compound, included at three levels, were mixed with the sample powder and extracted with $95 \%$ methanol containing the internal standard $(50 \mathrm{ppm}$ amiodarone hydrochloride). For comparison, the blank sample was prepared by spiking with $95 \%$ methanol containing the

Table 3. Robustness of Marker Compounds According to Columns, Temperatures and Flow Rates $(n=3)$

\begin{tabular}{|c|c|c|c|c|c|c|}
\hline Marker & \multicolumn{2}{|c|}{ Analytical condition } & $\begin{array}{l}\text { Theoretical plate } \\
(N)\end{array}$ & $\begin{array}{l}\text { Capacity factor } \\
\qquad\left(\boldsymbol{k}^{\prime}\right)\end{array}$ & $\begin{array}{l}\text { Separation factor } \\
\qquad(\alpha)\end{array}$ & $\begin{array}{c}\text { Resolution } \\
(\boldsymbol{R s})\end{array}$ \\
\hline \multirow{9}{*}{1} & \multirow{3}{*}{ Column $^{a)}$} & Aegispak & $9397 \pm 67$ & $3.63 \pm 0.01$ & $1.11 \pm 0.00$ & $1.94 \pm 0.02$ \\
\hline & & Gemini & $4724 \pm 98$ & $3.42 \pm 0.05$ & $1.09 \pm 0.01$ & $1.69 \pm 0.19$ \\
\hline & & Shiseido & $5218 \pm 37$ & $2.60 \pm 0.03$ & $1.11 \pm 0.00$ & $1.74 \pm 0.04$ \\
\hline & \multirow{3}{*}{$\begin{array}{c}\text { Temperature } \\
\left({ }^{\circ} \mathrm{C}\right)\end{array}$} & 25 & $9397 \pm 67$ & $3.63 \pm 0.01$ & $1.11 \pm 0.00$ & $1.94 \pm 0.02$ \\
\hline & & 30 & $9329 \pm 37$ & $3.49 \pm 0.01$ & $1.10 \pm 0.00$ & $2.59 \pm 0.03$ \\
\hline & & 35 & $9899 \pm 50$ & $3.32 \pm 0.01$ & $1.10 \pm 0.00$ & $2.13 \pm 0.07$ \\
\hline & \multirow{3}{*}{$\begin{array}{l}\text { Flow rate } \\
(\mathrm{mL} / \mathrm{min})\end{array}$} & 0.8 & $10443 \pm 31$ & $3.21 \pm 0.03$ & $1.10 \pm 0.00$ & $2.42 \pm 0.49$ \\
\hline & & 1.0 & $9397 \pm 67$ & $3.63 \pm 0.01$ & $1.11 \pm 0.00$ & $1.94 \pm 0.02$ \\
\hline & & 1.2 & $7164 \pm 31$ & $3.94 \pm 0.02$ & $1.11 \pm 0.00$ & $2.29 \pm 0.02$ \\
\hline \multirow{9}{*}{2} & \multirow{3}{*}{ Column } & Aegispak & $4261 \pm 98$ & $4.12 \pm 0.01$ & $1.17 \pm 0.00$ & $2.87 \pm 0.29$ \\
\hline & & Gemini & $4583 \pm 25$ & $3.83 \pm 0.01$ & $1.16 \pm 0.00$ & $2.46 \pm 0.03$ \\
\hline & & Shiseido & $3842 \pm 163$ & $3.00 \pm 0.03$ & $1.19 \pm 0.00$ & $3.24 \pm 0.01$ \\
\hline & \multirow{3}{*}{$\begin{array}{l}\text { Temperature } \\
\left({ }^{\circ} \mathrm{C}\right)\end{array}$} & 25 & $4261 \pm 98$ & $4.12 \pm 0.01$ & $1.17 \pm 0.00$ & $2.87 \pm 0.29$ \\
\hline & & 30 & $4773 \pm 22$ & $3.95 \pm 0.01$ & $1.16 \pm 0.00$ & $4.11 \pm 0.03$ \\
\hline & & 35 & $6601 \pm 64$ & $3.75 \pm 0.02$ & $1.15 \pm 0.00$ & $3.43 \pm 0.13$ \\
\hline & \multirow{3}{*}{$\begin{array}{l}\text { Flow rate } \\
(\mathrm{mL} / \mathrm{min})\end{array}$} & 0.8 & $4506 \pm 102$ & $3.63 \pm 0.03$ & $1.15 \pm 0.00$ & $3.11 \pm 0.14$ \\
\hline & & 1.0 & $4261 \pm 98$ & $4.12 \pm 0.01$ & $1.17 \pm 0.00$ & $2.87 \pm 0.29$ \\
\hline & & 1.2 & $6994 \pm 54$ & $4.47 \pm 0.02$ & $1.17 \pm 0.00$ & $3.01 \pm 0.01$ \\
\hline \multirow{9}{*}{3} & \multirow{3}{*}{ Column } & Aegispak & $7860 \pm 35$ & $4.97 \pm 0.02$ & $1.58 \pm 0.00$ & $16.84 \pm 2.27$ \\
\hline & & Gemini & $4803 \pm 47$ & $4.62 \pm 0.01$ & $1.49 \pm 0.00$ & $9.55 \pm 0.07$ \\
\hline & & Shiseido & $7549 \pm 15$ & $3.77 \pm 0.04$ & $1.76 \pm 0.01$ & $18.97 \pm 0.62$ \\
\hline & \multirow{3}{*}{$\begin{array}{c}\text { Temperature } \\
\left({ }^{\circ} \mathrm{C}\right)\end{array}$} & 25 & $12860 \pm 35$ & $4.97 \pm 0.02$ & $1.58 \pm 0.00$ & $16.84 \pm 2.27$ \\
\hline & & 30 & $11928 \pm 39$ & $4.72 \pm 0.01$ & $1.65 \pm 0.00$ & $20.68 \pm 0.24$ \\
\hline & & 35 & $14107 \pm 127$ & $4.47 \pm 0.01$ & $1.72 \pm 0.00$ & $24.64 \pm 0.51$ \\
\hline & \multirow{3}{*}{$\begin{array}{l}\text { Flow rate } \\
(\mathrm{mL} / \mathrm{min})\end{array}$} & 0.8 & $7158 \pm 26$ & $4.34 \pm 0.04$ & $1.50 \pm 0.01$ & $12.99 \pm 1.93$ \\
\hline & & 1.0 & $7860 \pm 35$ & $4.97 \pm 0.02$ & $1.58 \pm 0.00$ & $16.84 \pm 2.27$ \\
\hline & & 1.2 & $4315 \pm 12$ & $5.41 \pm 0.03$ & $1.69 \pm 0.01$ & $15.06 \pm 0.20$ \\
\hline \multirow{9}{*}{4} & \multirow{3}{*}{ Column } & Aegispak & $37049 \pm 22$ & $10.78 \pm 0.02$ & $1.29 \pm 0.00$ & $10.92 \pm 0.09$ \\
\hline & & Gemini & $15809 \pm 45$ & $9.78 \pm 0.02$ & $1.21 \pm 0.00$ & $5.98 \pm 0.03$ \\
\hline & & Shiseido & $43661 \pm 21$ & $9.76 \pm 0.03$ & $1.29 \pm 0.00$ & $9.67 \pm 0.15$ \\
\hline & \multirow{3}{*}{$\begin{array}{c}\text { Temperature } \\
\left({ }^{\circ} \mathrm{C}\right)\end{array}$} & 25 & $67049 \pm 22$ & $10.78 \pm 0.02$ & $1.29 \pm 0.00$ & $10.92 \pm 0.09$ \\
\hline & & 30 & $79908 \pm 27$ & $10.67 \pm 0.01$ & $1.27 \pm 0.00$ & $12.48 \pm 0.24$ \\
\hline & & 35 & $75155 \pm 26$ & $10.54 \pm 0.01$ & $1.25 \pm 0.00$ & $11.73 \pm 0.26$ \\
\hline & \multirow{3}{*}{$\begin{array}{l}\text { Flow rate } \\
(\mathrm{mL} / \mathrm{min})\end{array}$} & 0.8 & $49874 \pm 17$ & $9.35 \pm 0.01$ & $1.32 \pm 0.00$ & $13.05 \pm 0.52$ \\
\hline & & 1.0 & $37049 \pm 22$ & $10.78 \pm 0.02$ & $1.29 \pm 0.00$ & $10.92 \pm 0.09$ \\
\hline & & 1.2 & $43237 \pm 101$ & $12.15 \pm 0.02$ & $1.26 \pm 0.00$ & $9.20 \pm 0.13$ \\
\hline \multirow{9}{*}{5} & \multirow{3}{*}{ Column } & Aegispak & $34151 \pm 10$ & $14.20 \pm 0.04$ & $1.07 \pm 0.00$ & $3.18 \pm 0.14$ \\
\hline & & Gemini & $27621 \pm 63$ & $12.01 \pm 0.03$ & $1.06 \pm 0.00$ & $2.26 \pm 0.02$ \\
\hline & & Shiseido & $21748 \pm 94$ & $12.89 \pm 0.06$ & $1.09 \pm 0.02$ & $3.84 \pm 0.71$ \\
\hline & \multirow{3}{*}{$\begin{array}{l}\text { Temperature } \\
\left({ }^{\circ} \mathrm{C}\right)\end{array}$} & 25 & $44151 \pm 10$ & $14.20 \pm 0.04$ & $1.07 \pm 0.00$ & $3.18 \pm 0.14$ \\
\hline & & 30 & $46216 \pm 24$ & $13.81 \pm 0.01$ & $1.07 \pm 0.00$ & $3.06 \pm 0.05$ \\
\hline & & 35 & $47405 \pm 25$ & $13.43 \pm 0.01$ & $1.06 \pm 0.00$ & $3.00 \pm 0.06$ \\
\hline & \multirow{3}{*}{$\begin{array}{l}\text { Flow rate } \\
(\mathrm{mL} / \mathrm{min})\end{array}$} & 0.8 & $38844 \pm 51$ & $12.64 \pm 0.03$ & $1.07 \pm 0.00$ & $3.22 \pm 0.08$ \\
\hline & & 1.0 & $44151 \pm 10$ & $14.20 \pm 0.04$ & $1.07 \pm 0.00$ & $3.18 \pm 0.14$ \\
\hline & & 1.2 & $38514 \pm 12$ & $15.51 \pm 0.05$ & $1.07 \pm 0.00$ & $2.79 \pm 0.09$ \\
\hline
\end{tabular}

Rutin (1), isoquercetin (2), 3,5-dicaffeoylquinic acid (3), tussilagone (4), and tussilagonone (5). a) YoungJinBioChrom Aegispak C18-L (250 mm $\times 4.6 \mathrm{~mm}, 5 \mu \mathrm{m}$ ), Phenomenex Gemini C18 110A $(250 \mathrm{~mm} \times 4.6 \mathrm{~mm}, 5 \mu \mathrm{m})$, and Shiseido C18 $(250 \mathrm{~mm} \times 4.6 \mathrm{~mm}, 5 \mu \mathrm{m})$. 
Table 4. Stability of Marker Compounds

\begin{tabular}{|c|c|c|c|c|c|c|c|c|c|}
\hline \multirow{2}{*}{ Compounds } & \multirow{2}{*}{$\begin{array}{c}\text { Temperature } \\
\left({ }^{\circ} \mathrm{C}\right)\end{array}$} & \multicolumn{6}{|c|}{ Day (\%) } & \multirow{2}{*}{$\begin{array}{l}\text { Mean } \\
(n=6)\end{array}$} & \multirow{2}{*}{$\begin{array}{c}\text { R.S.D. }{ }^{a)} \\
(\%)\end{array}$} \\
\hline & & 0 & 1 & 3 & 7 & 15 & 30 & & \\
\hline \multirow{2}{*}{1} & 4 & $100^{b)}$ & 98.51 & 101.99 & 99.33 & 101.11 & 97.12 & 99.68 & 1.77 \\
\hline & 25 & 100 & 96.86 & 95.61 & 98.56 & 97.96 & 101.43 & 98.40 & 2.14 \\
\hline \multirow{2}{*}{2} & 4 & 100 & 98.29 & 100.71 & 97.44 & 99.77 & 95.57 & 98.63 & 1.95 \\
\hline & 25 & 100 & 95.25 & 95.97 & 97.29 & 98.22 & 100.17 & 97.82 & 2.08 \\
\hline \multirow{2}{*}{3} & 4 & 100 & 99.16 & 91.26 & 86.15 & 84.98 & 83.70 & 90.87 & 7.95 \\
\hline & 25 & 100 & 87.19 & 84.86 & 79.49 & 66.18 & 66.23 & 80.66 & 16.20 \\
\hline \multirow{2}{*}{4} & 4 & 100 & 98.45 & 101.73 & 99.48 & 98.72 & 95.76 & 99.02 & 2.00 \\
\hline & 25 & 100 & 98.04 & 95.72 & 98.12 & 100.99 & 99.81 & 98.78 & 1.91 \\
\hline \multirow{2}{*}{5} & 4 & 100 & 98.28 & 97.95 & 99.19 & 95.74 & 95.02 & 97.70 & 1.99 \\
\hline & 25 & 100 & 95.15 & 95.72 & 98.11 & 98.11 & 95.81 & 97.15 & 1.94 \\
\hline
\end{tabular}

Rutin (1), isoquercetin (2), 3,5-dicaffeoylquinic acid (3), tussilagone (4), and tussilagonone (5). $a$ ) R.S.D. (\%) =S.D./mean $\times 100$. $b$ ) Percentage of peak ratio of marker compound to internal standard according to days.

internal standard (50 ppm amiodarone hydrochloride). The quantitation of each component was subsequently achieved from the corresponding calibration curves. The recoveries of the all components were between $93.9 \%$ and $106.7 \%(n=6)$, with a relative standard deviation (R.S.D.) $<4.4 \%$.

The robustness was determined in order to evaluate the reliability of the established HPLC method. All parameters were maintained so there would be no interference with the other peaks for the Farfarae Flos. The experimental conditions, such as column temperature, column species and mobile phases, were purposefully altered. Evaluation was carried out on the theoretical plate $(\boldsymbol{N})$, capacity factor $\left(\boldsymbol{k}^{\prime}\right)$, separation factor $(\boldsymbol{\alpha})$ and resolution $(\boldsymbol{R} \boldsymbol{s})$. To evaluate the suitability of three different columns, YoungJinBioChrom Aegispak, Phenomenex Gemini, and Shiseido, the performances were compared with regard to four analytical factors $\left(\boldsymbol{N}, \boldsymbol{k}^{\prime}, \boldsymbol{\alpha}\right.$, and $\left.\boldsymbol{R} \boldsymbol{s}\right)$ at the column temperature of $25^{\circ} \mathrm{C}$. The result showed no large differences in the factors depending on the column. Comparison of the factors was then done for the Aegispak column at three different column temperatures, 25,30 , and $35^{\circ} \mathrm{C}$. Similarly, the results indicated that great variation in the factors did not occur depending on the column temperature $\left(25,30,35^{\circ} \mathrm{C}\right)$. Further comparison of three different flow rates, 0.8, 1.0 and $1.2 \mathrm{~mL} / \mathrm{min}$, for the Aegispak column at $25^{\circ} \mathrm{C}$ also revealed that the four analytical factors did not differ greatly depending on the flow rates $(0.8,1.0,1.2 \mathrm{~mL} / \mathrm{min})$. Optimization was also carried out by changing the chromatographic parameters, but no great variation was observed. Therefore, this experimental condition was sufficiently robust (Table 3 ).

The sample stability test was carried out with a standard mixture solution at $0,1,3,7,15$, and $30 \mathrm{~d}$. During this period, the solution was stored in lightless conditions at room temperature $\left(25^{\circ} \mathrm{C}\right)$ and $4^{\circ} \mathrm{C}$. The recovery rate of 3,5-dicaffeoylquinic acid (3) was found to drop below $90 \%$ after $1 \mathrm{~d}$ at room temperature; Even a recovery of this compound was confirmed as $91.3 \%$ after $3 \mathrm{~d}$ at $4^{\circ} \mathrm{C}$, respectively. After $30 \mathrm{~d}$, the recovery rates were down to 66.23 and $83.70 \%$ at room temperature and $4^{\circ} \mathrm{C}$, respectively. At $4^{\circ} \mathrm{C}$, this compound demonstrated $99.2 \%$ recovery for $24 \mathrm{~h}$. Thus, the content of this compound in the extract should be measured within $24 \mathrm{~h}$ of storage at $4^{\circ} \mathrm{C}$. The other marker compounds demonstrated stability for $30 \mathrm{~d}$, as shown in Table 4 .
Sample Analysis The established HPLC/PDA analytical method was applied for the simultaneous determination of five standard compounds, rutin (1), isoquercetin (2), 3,5-dicaffeoylquinic acid (3), tussilagone (4), and tussilagonone (5), in Farfarae Flos [Korean products (K01-K06), and Chinese products (C7-C62)]. The quantity of each compound present in the samples was determined, and the results are summarized in Table 5. Each sample was analyzed in triplicate to ensure reproducibility of the quantitative result. The average contents of rutin $(0.090 \pm 0.055 \%)$ and tussilagonone $(0.021 \pm 0.009 \%)$ in the Chinese Farfarae Flos samples were not significantly different from those of rutin $(0.078 \pm 0.015 \%)$ and tussilagonone $(0.019 \pm 0.015 \%)$ in the Korean Farfarae Flos samples. However, the average isoquercetin $(0.046 \pm 0.034 \%)$ and 3,5-dicaffeoylquinic acid $(0.537 \pm 0.146 \%)$ content in the Chinese Farfarae Flos were lower than those of isoquercetin $(0.086 \pm 0.037 \%)$ and 3,5-dicaffeoylquinic acid $(0.696 \pm 0.117 \%)$ in Korean Farfarae Flos. Interestingly, the average tussilagone content in Chinese Farfarae Flos was found to be $0.101 \pm 0.029 \%$, whereas little or no detection was observed for the Korean samples (Table 6). This result should be confirmed with more data from a greater number of Korean samples.

Pattern Recognition Analysis A pattern recognition analysis method using the contents of five markers [rutin (1), isoquercetin (2), 3,5-dicaffeoylquinic acid (3), tussilagone (4), and tussilagonone (5)] was carried out for quality evaluation of the Farfarae Flos. All samples were clustered into two groups by hierarchical cluster analysis in SPDD, according to geographic origin: Chinese Farfarae Flos (group A; C07-C62), and Korean Farfarae Flos (group B; K01-K06) (Fig. 3). Thus, these pattern analysis results could be used for the quality control of Farfarae Flos samples.

\section{Conclusion}

An analytical method for Farfarae Flos by HPLC was developed for the separation of five marker compounds [rutin (1), isoquercetin (2), 3,5-dicaffeoylquinic acid (3), tussilagone (4), and tussilagonone (5)] using amiodarone hydrochloride as an internal standard. HPLC separation was carried out using the Aegispak C18-L $(250 \times 4.6 \mathrm{~mm}, 5 \mu \mathrm{m})$ column with a three-step solvent system by PDA. The results suggested that the established HPLC/PDA method was suitable for the quantitative 


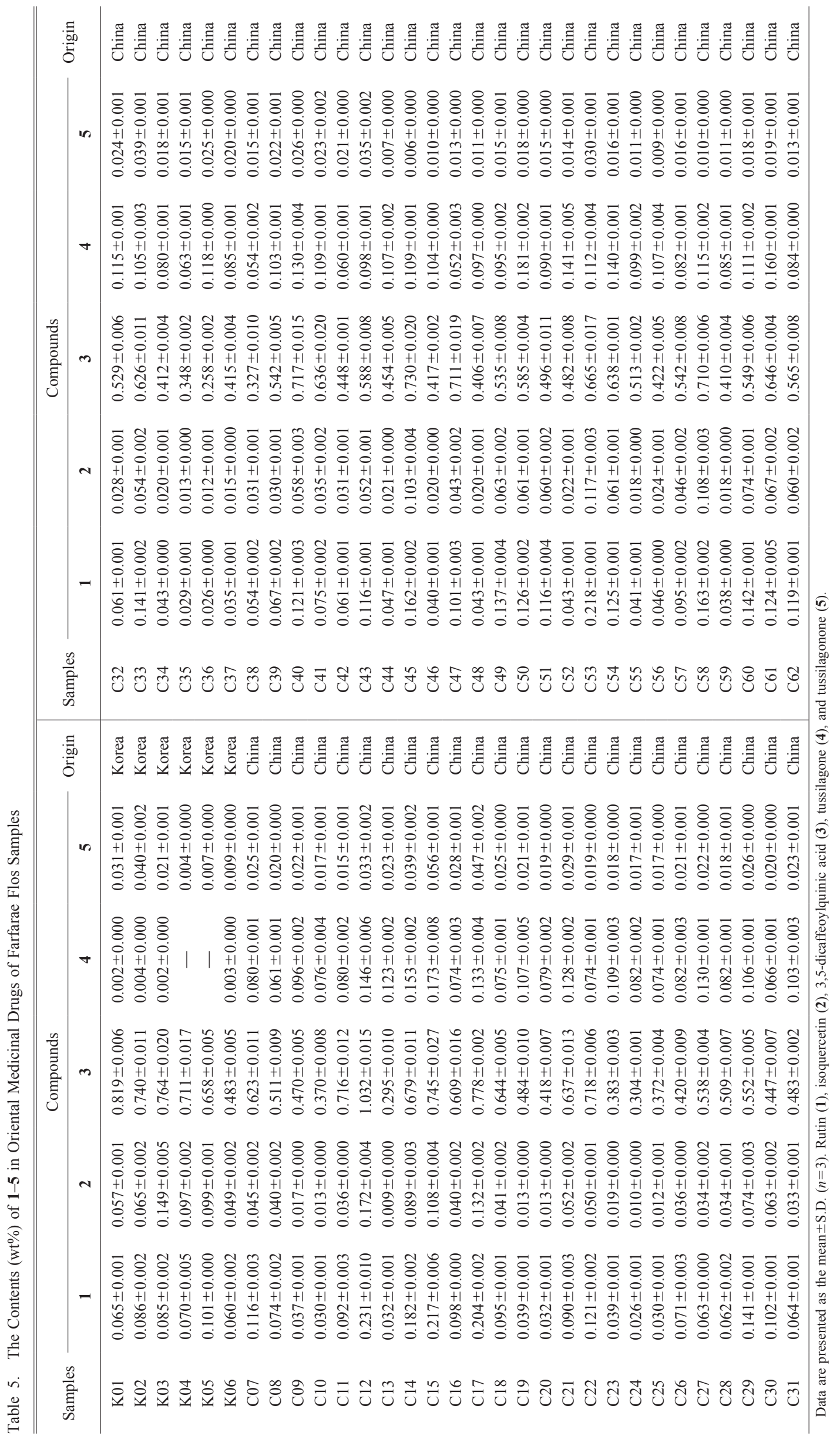




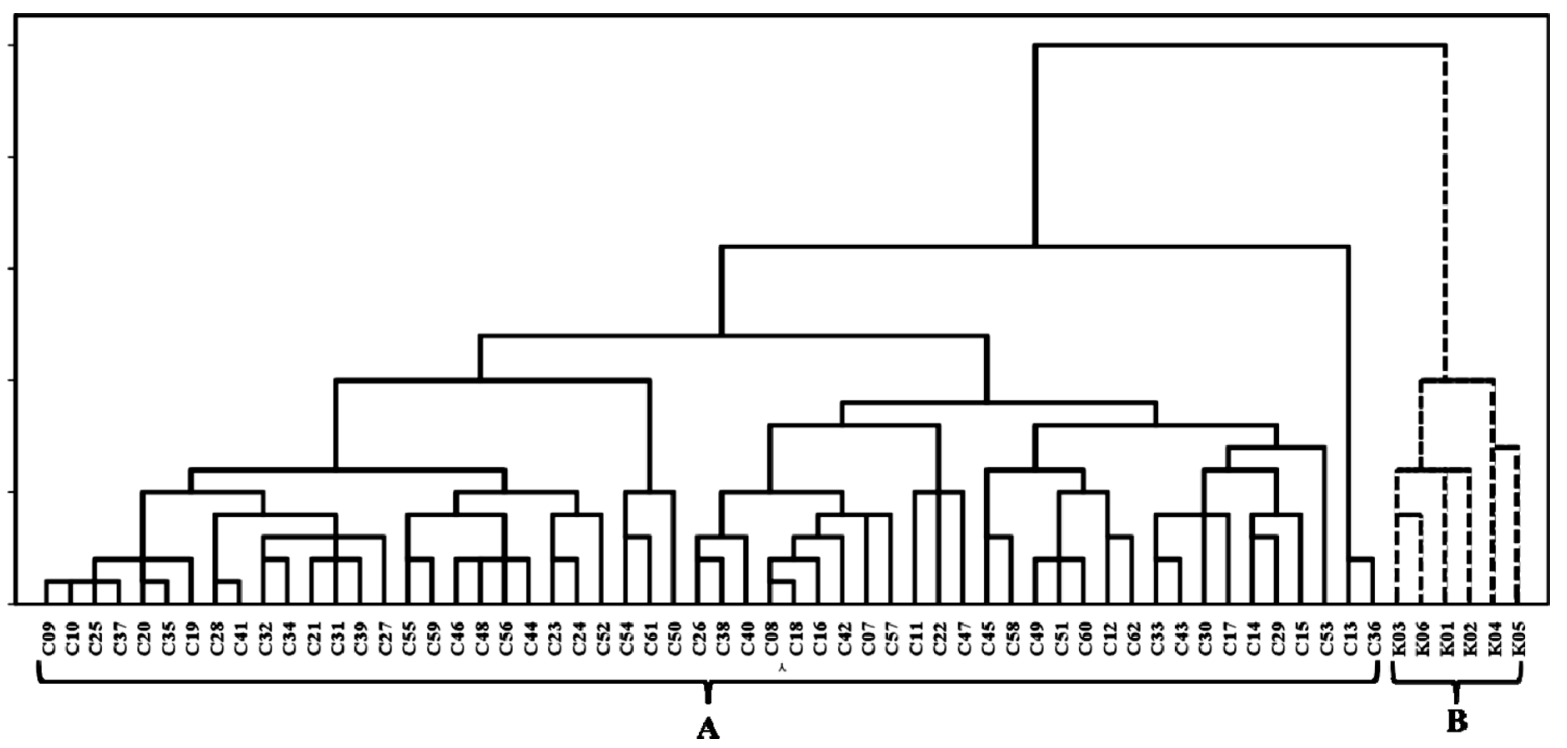

Fig. 3. Hierarchical Cluster Analysis by SPSS of 62 Samples Based on the Contents of Marker Compounds

A: Chinese Farfarae Flos (C07-C62), B: Korean Farfarae Flos (K01-K06).

Table 6. The Average Content (wt $\%)$ of Marker Compounds $(n=3)$

\begin{tabular}{cccc}
\hline \hline Compounds & $\begin{array}{c}\text { Chinese Farfarae } \\
\text { Flos samples } \\
(n=56)\end{array}$ & $\begin{array}{c}\text { Korean Farfarae } \\
\text { Flos samples } \\
(n=6)\end{array}$ & $\begin{array}{c}\text { Total } \\
(n=62)\end{array}$ \\
\hline \multirow{2}{*}{$\mathbf{1}$} & $0.090 \pm 0.055$ & $0.078 \pm 0.015$ & $0.089 \pm 0.052$ \\
$(0.026-0.231)$ & $(0.060-0.101)$ & $(0.026-0.231)$ \\
\hline \multirow{2}{*}{$\mathbf{2}$} & $0.046 \pm 0.034$ & $0.086 \pm 0.037$ & $0.050 \pm 0.036$ \\
& $(0.009-0.172)$ & $(0.049-0.149)$ & $(0.009-0.172)$ \\
\hline \multirow{3}{*}{$\mathbf{4}$} & $0.537 \pm 0.146$ & $0.696 \pm 0.117$ & $0.552 \pm 0.150$ \\
& $(0.258-1.032)$ & $(0.483-0.819)$ & $(0.258-1.032)$ \\
\hline \multirow{2}{*}{$\mathbf{5}$} & $0.101 \pm 0.029$ & $0.002 \pm 0.002$ & $0.092 \pm 0.041$ \\
& $(0.052-0.181)$ & $(0.000-0.004)$ & $(0.000-0.181)$ \\
\hline & $0.021 \pm 0.009$ & $0.019 \pm 0.015$ & $0.021 \pm 0.010$ \\
& $(0.006-0.056)$ & $(0.004-0.040)$ & $(0.004-0.056)$ \\
\hline
\end{tabular}

Rutin (1), isoquercetin (2), 3,5-dicaffeoylquinic acid (3), tussilagone (4), and tussilagonone (5).

analysis and quality control of Farfarae Flos. Based on the detected quantities of the five standard compounds, the Korean and the Chinese Farfarae Flos samples were distinguished by pattern analysis. The 3,5-dicaffeoylquinic acid content was the highest, but this compound was confirmed to undergo rapid decomposition. Large variation was observed in the tussilagonone content, ranging from 0.004 to $0.056 \%$. Therefore, 3,5-dicaffeoylquinic acid and tussilagonone were not suitable as marker compounds. Consequently, we suggest that the regulation of tussilagone, rutin and isoquercetin as marker compounds is more reasonable compared with the use of only one marker compound (tussilagone), as currently employed in the C.P. In addition, the tussilagone content in the Korean Farfarae Flos was low or could not be detected. It is considered that $T$. farfara is not suitable for cultivation in Korea because of climatic conditions. Actually, all Farfarae Flos circulating in the Korean market is currently imported from China.

Acknowledgment This work was supported by a Grant from the National Center for Standardization of Herbal Medi- cine funded by the Ministry of Food and Drug Safety, Republic of Korea (09112KFDA890).

Conflict of Interest The authors declare no conflict of interest.

\section{References}

1) Islam M. N., Lee S. K., Jeong S. Y., Kim D. H., Jin C., Yoo H. H., Bull. Korean Chem. Soc., 30, 137-144 (2009).

2) Yaoita Y., Kamazawa H., Kikuchi M., Chem. Pharm. Bull., 47, 705-707 (1999).

3) Park H. R., Yoo M. Y., Seo J. H., Kim I. S., Kim N. Y., Kang J. Y., Cui L., Lee C. S., Lee C. H., Lee H. S., J. Agric. Food Chem., 56, 10493-10497 (2008).

4) Kim M. R., Lee J. Y., Lee H. H., Aryal D. K., Kim Y. G., Kim S. K., Woo E. R., Kang K. W., Food Chem. Toxicol., 44, 1299-1307 (2006).

5) Kokoska L., Polesny Z., Rada V., Nepovim A., Vanek T., J. Ethnopharmacol., 82, 51-53 (2002).

6) Lim H. J., Lee H. S., Ryu J. H., Arch. Pharm. Res., 31, 645-652 (2008)

7) Lee M. R., Cha M. R., Jo K. J., Yoon M. Y., Park H. R., Food Sci. Biotechnol., 17, 308-312 (2008).

8) Nanjing University of Chinese Medicine, "Dictionary of Chinese Herbal Medicine," Shanghai Science and Technology Publishing House, Shanghai, 2006, p. 3195.

9) The Pharmacopoeia Commission of the PRC, "Pharmacopoeia of People's Republic of China," Chemical Industry Press, Beijing, 2010, pp. 3194-3195.

10) Korea Food and Drug Administration, "Korean Pharmacopoeia," Part II, Shinil Books, Seoul, 2014, p. 16.

11) Liu Y., Ye Y. L., Wan D. G., Wang Y., Li B., Lishizhen Med. Mater. Med. Res., 18, 2722-2723 (2007).

12) Lu P., Lan Y., Hou J., Li C., Niu Y., China Pharm., 11, 1307-1308 (2008).

13) Liu X., Cao J., Chin. J. Pharm. Anal., 29, 134-136 (2009).

14) Wu D., Wang Z., Zhang M., Chin. J. Chin. Mater. Med., 35, 2722 2725 (2010).

15) Zhang W. Y., Yang T. S., Wang X. P., Dang W. X., Chin. J. Pharm. Anal., 28, 2106-2108 (2008).

16) Ma Z. J., Dong H. H., Li Z. Y., Zhou Y. Z., Qin X. M., Guo X. Q., Zhang L. Z., Chin. Tradit. Herbal Drugs, 40, 1305-1308 (2009). 